EPJ Web of Conferences 37, 01010 (2012)

DOI: $10.1051 /$ epjconf/20123701010

(C) Owned by the authors, published by EDP Sciences, 2012

\title{
Kaon Physics at CERN - Recent Results from the NA48/2 and NA62 Experiments
}

\author{
Andreas Winhart $^{\mathrm{a}}$ for the NA48/2 and NA62 collaborations \\ Institut für Physik, Johannes-Gutenberg-Universität, 55099 Mainz, Germany
}

\begin{abstract}
This letter presents recent results from the kaon experiments NA48/2 and its successor NA62 at CERN. With samples of $2.5 \times 10^{6} K_{\mu 3}^{ \pm}$and $4.0 \times 10^{6} K_{e 3}^{ \pm}$events collected by NA48/2 in 2004, precise measurements of the form factors were performed. The Chiral Perturbation Theory was tested through a new study of $K^{ \pm} \rightarrow \pi^{ \pm} \gamma \gamma$ decays based on data taken with minimal trigger conditions. Using the beam line and detector of NA48/2 in the years 2007-2008, NA62 performed a precison measurement of the helicitysuppressed ratio of the charged kaon leptonic decay rates $K^{ \pm} \rightarrow e^{+} v$ and $K^{ \pm} \rightarrow \mu^{+} v$. Using the full data set, which is about ten times larger than the previous world data sample, the total uncertainty on this ratio is only $0.4 \%$, representing a precision test of $\mu-e$ lepton universality. Finally, the main stage of the NA62 experiment is presented. The planned measurement of the ultra rare decay $K^{+} \rightarrow \pi^{+} v \bar{v}$ at $10 \%$ precision will provide a powerful test of the Standard Model. The experimental challenges to reach this goal require new detectors with exceptional performances.
\end{abstract}

Key words. kaons - rare decays - form factors - ChPT - new physics - lepton universality

\section{Introduction}

Kaon physics is still a very active field and provides important contributions to the understanding of fundamental processes and structures. The experimental precision is outstanding, allowing to study extremely rare processes with good sensitivity to possible contributions from New Physics.

In 2003-2004, the NA48/2 experiment at the CERN SPS collected the world largest sample of charged kaon decays. The main goal of this data taking was the search for direct CP violating charge asymmetries in decays to three pions in the final state. In addition, many other precision studies have been performed on this multipurpose data set, taking advantage of the highly symmetric experimental conditions. In 2007-2008, the NA62 collaboration used the NA48/2 experimental setup at somewhat modified data taking conditions ( $R$ phase) to measure the ratio of the decay rates of the leptonic decays $R_{K}=\Gamma\left(K^{ \pm} \rightarrow e^{ \pm} v\right) / \Gamma\left(K^{ \pm} \rightarrow \mu^{ \pm} v\right)$. The final phase of the NA62 experiment with new beam line and detector is expected to start in 2014. The main goal will be the measurement of the $K^{+} \rightarrow \pi^{+} v \bar{v}$ decay rate.

\section{Data Taking, Beam and Detector}

The beam line was designed to deliver simultaneous $K^{+}$and $K^{-}$beams with a narrow momentum spread produced by $400 \mathrm{GeV} / c$ protons from the CERN SPS impinging on a beryllium target. After passing a set of collimators, the kaon beams with a central momentum of $60 \mathrm{GeV} / c$ in 2003/2004 and $74 \mathrm{GeV} / \mathrm{c}$ in $2007 / 2008$ entered a fiducial decay volume in a $114 \mathrm{~m}$ long cylindrical vacuum tank,

\footnotetext{
a e-mail: awinhart@uni-mainz.de
} 
which was followed by the main detector. The subdetectors relevant for the presented analyses are: a) A magnetic spectrometer consisting of four drift chambers (DCHs) with a central dipole magnet and four views per chamber, used to measure the momenta of charged particles. b) A plastic scintillator hodoscope (HOD) with good time resolution to provide fast trigger signals. c) The liquid krypton electromagnetic calorimeter ( $\mathrm{LKr}$ ) used for $\gamma$ detection and particle identification. It's a quasi homogeneous ionization chamber, 27 radiation lengths deep, with $7 \mathrm{~m}^{3}$ of krypton as active medium and transversally segmented into 13248 projective cells $\left(2 \times 2 \mathrm{~cm}^{2}\right.$ each). The NA48 detector is described in detail in [1].

The analyses presented in this letter are based on two data taking periods with minimum bias trigger configurations: a special three days run in 2004 (NA48/2), and four months in 2007 (NA62).

\section{Precision Measurement of $K_{l 3}^{ \pm}$Form Factors}

Semileptonic kaon decays $\left(K_{l 3}, l=\mu, e\right)$ provide the most accurate and theoretically cleanest way to extract the CKM matrix element $\left|V_{u s}\right|$ [2]. Their hadronic matrix element is described by two dimensionless form factors $f_{ \pm}(t)$, functions of the 4-momentum transfer $t$ to the lepton system. The form factors are mandatory input parameters to the phase space integrals of these decays to determine $\left|V_{u s}\right|$.

Usually, form factors are re-formulated to express the vector $f_{+}(t)$ and scalar $f_{0}(t)$ exchange contributions, and $f_{0}(t)$ can be described as a linear combination of $f_{ \pm}(t)$ :

$$
f_{0}(t)=f_{+}(t)+\frac{t}{m_{K}^{2}-m_{\pi}^{2}} f_{-}(t)
$$

with $f_{+}(0)=f_{-}(0)$ by construction. Being proportional to the lepton mass squared, the contribution of $f_{-}(t)$ can be measured only in $K_{\mu 3}$ decays. As $f_{+}(0)$ cannot be mesured directly, it is common to normalize all form factors to it: $\bar{f}_{+}(t)=f_{+}(t) / f_{+}(0)$ and $\bar{f}_{0}(t)=f_{0}(t) / f_{+}(0)$.

To describe the form factors, different parametrizations are available. The most common ones, and reported in this letter, are

a) The Taylor expansion, called quadratic parametrization:

$$
\bar{f}_{+, 0}(t)=1+\lambda_{+, 0}^{\prime} \frac{t}{m_{\pi}^{2}}+\frac{1}{2} \lambda_{+, 0}^{\prime \prime}\left(\frac{t}{m_{\pi}^{2}}\right)^{2},
$$

where $\lambda^{\prime}$ and $\lambda^{\prime \prime}$ are the slope and curvature of the form factors, respectively. To reduce the number of parameters and to add a physical motivation, it is also common to use the

b) Pole parametrization

$$
\bar{f}_{+, 0}(t)=\frac{m_{V, S}^{2}}{m_{V, S}^{2}-t},
$$

where the exchange of vector and scalar resonances $K^{*}$ with spin parity $1^{-} / 0^{+}$is assumed, and the corresponding pole masses $m_{V} / m_{S}$ are the only free parameters. The special run with minimum bias trigger conditions allowed a clean selection of the $K_{l 3}^{ \pm}$signal channels with very low background at per-mil level. After all cuts, the selected samples amount to $4.0 \times 10^{6} K_{e 3}^{ \pm}$and $2.5 \times 10^{6} K_{\mu 3}^{ \pm}$events. To

Table 1. Preliminary form factor fit results for the quadratic and the pole parametrization. For the combined result, statistical and systematic uncertainties were combined.

\begin{tabular}{c|c|c|c}
\hline Quadratic $\left(\times 10^{-3}\right)$ & $\lambda_{+}^{\prime}$ & $\lambda_{+}^{\prime \prime}$ & $\lambda_{\mathbf{0}}$ \\
\hline$K_{e 3}^{ \pm}$ & $27.2 \pm 0.7_{\text {stat }} \pm 1.1_{\text {syst }}$ & $0.7 \pm 0.3_{\text {stat }} \pm 0.4_{\text {syst }}$ & \\
$K_{\mu 3}^{ \pm}$ & $26.3 \pm 3.0_{\text {stat }} \pm 2.2_{\text {syst }}$ & $1.2 \pm 1.1_{\text {stat }} \pm 1.1_{\text {syst }}$ & $15.7 \pm 1.4_{\text {stat }} \pm 1.0_{\text {syst }}$ \\
combined & $27.0 \pm 1.1$ & $0.8 \pm 0.5$ & $16.2 \pm 1.0$ \\
\hline \hline Pole $\left(\mathrm{MeV} / c^{2}\right)$ & $\boldsymbol{m}_{V}$ & & $\boldsymbol{m}_{\boldsymbol{S}}$ \\
\hline$K_{e 3}^{ \pm}$ & $879 \pm 7_{\text {stat }} \pm 7_{\text {syst }}$ & & $1183 \pm 31_{\text {stat }} \pm 16_{\text {syst }}$ \\
$K_{\mu 3}^{ \pm}$ & $873 \pm 8_{\text {stat }} \pm 9_{\text {syst }}$ & & $1176 \pm 31$ \\
combined & $877 \pm 6$ & & \\
\hline
\end{tabular}



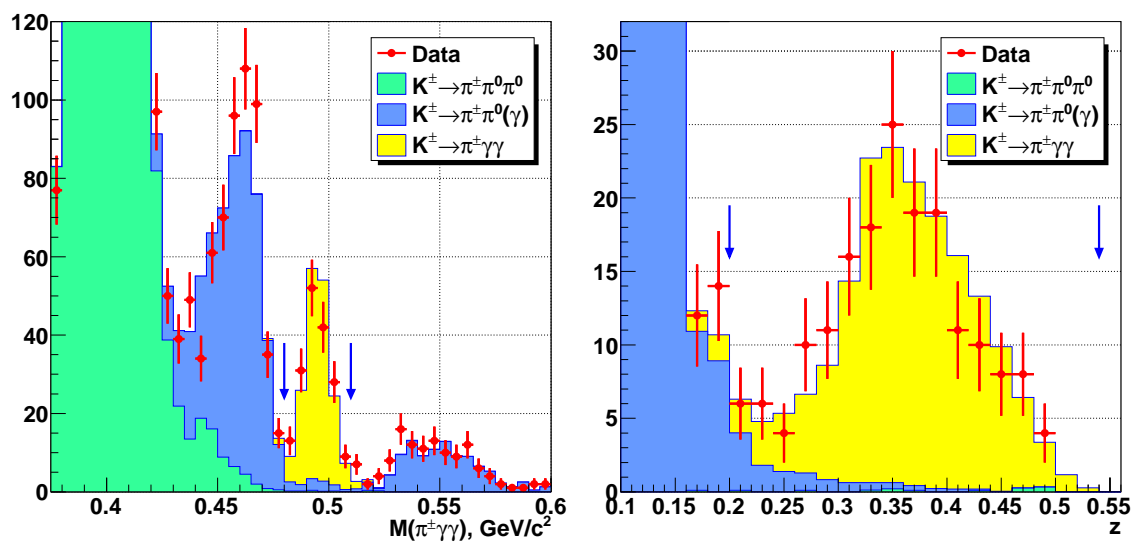

Fig. 1. Data distributions based on the NA62 run 2007. Left: Spectrum of the $\pi^{ \pm} \gamma \gamma$ invariant mass with MC expectations for signal and background. Right: Spectrum of $z=\left(m_{\gamma \gamma} / m_{K}\right)^{2}$ with MC for signal (best fit) and backgrounds. In both plots, the signal regions are indicated with arrows.

extract the form factors, a fit to the Dalitz plot density was performed. Before, the Dalitz plot had to be corrected for remaining background, detector acceptance and distortions induced by radiative effects. The obtained preliminary results are listed in table 1, being in good agreement with the measurements by other experiments. NA48/2, however, is the first experiment which measured both $K_{e 3}^{ \pm}$and $K_{\mu 3}^{ \pm}$, and it offers the combined result with the smallest uncertainty.

\section{A New Measurement of $K^{ \pm} \rightarrow \pi^{ \pm} \gamma \gamma$ Decays}

Radiative kaon decays offer a unique possibility to study Chiral Perturbation Theory (ChPT) with its ability to explain low energy processes. In particular, the $K^{ \pm} \rightarrow \pi^{ \pm} \gamma \gamma$ decay is of high interest since tree-level contributions at $O\left(p^{2}\right)$ vanish. At lowest non-trivial order $O\left(p^{4}\right)$, there are two noninterfering contributions: a) the pion and kaon loop amplitude depending on an unknown parameter $\hat{c}$, predicted to be of $O(1)$ and representing the total contribution of the counterterms, and b) the pole amplitude [3]. The loop leads to a characteristic signature in the invariant diphoton mass spectrum, exhibiting a cusp at the $\pi^{+} \pi^{-}$threshold, $m_{\gamma \gamma}=2 m_{\pi^{+}}$. The parameter $\hat{c}$ fixes the value of the branching ratio and the $m_{\gamma \gamma}$ spectrum shape. Higher order $O\left(p^{6}\right)$ unitarity corrections from $K^{ \pm} \rightarrow 3 \pi^{ \pm}$decays are predicted to modify the decay spectrum significantly, increasing the BR at low $\hat{c}$ and, in particular, resulting in a non-zero rate at $m_{\gamma \gamma} \rightarrow 0$ [4]. The only published experimental result on $K^{ \pm} \rightarrow \pi^{ \pm} \gamma \gamma$ is that of 31 candidates ( $K^{+}$decays) in the large diphoton mass region by the BNL E787 experiment [5].

The new measurements presented here are based on both sets of minimum bias data from NA48/2 (2004) and NA62 (2007). The latter sample has been collected with a set of downscaled trigger conditions with an effective downscaling factor of $\sim 20$. Signal events were selected in the region $z=\left(m_{\gamma \gamma} / m_{K}\right)^{2}>0.2$ to minimize the background from $K^{ \pm} \rightarrow \pi^{+} \pi^{0}$. After all cuts, 147 (175) $\pi^{ \pm} \gamma \gamma$ signal candidates were selected in the 2004 (2007) data sets, with backgrounds contributions of $12 \%$ (7\%) from $K^{ \pm} \rightarrow \pi^{ \pm} \pi^{0}\left(\pi^{0}\right)(\gamma)$ decays with merged photon clusters in the LKr calorimeter.

Fig. 1 shows data distributions based on the 2007 data sample: the $\pi^{ \pm} \gamma \gamma$ mass spectrum (left) and the spectrum of the $z$ kinematic variable (right), where the cusp-like behaviour at the two-pion threshold $(z \approx 0.32)$ is clearly visible, as predicted by the ChPT. The values of the $\hat{c}$ parameter have been determined by performing likelihood fits to the data. The preliminary results of the fits are shown in table 2; they are in agreement with the earlier BNL results. The uncertainties are dominated by the statistical ones; the systematic errors mainly come from the uncertainties on the background estimates. The combination of the 2004 and 2007 results takes into account the large positive correlation of their systematic uncertainties. 
Table 2. The preliminary results of the fits to the diphoton mass spectra with the ChPT parametrizations given in [4]. The quoted BR values correspond to the full kinematic range.

\begin{tabular}{c|c|c|c}
\hline & $\mathbf{2 0 0 4}(\mathbf{N A 4 8} / 2)$ & $\mathbf{2 0 0 7}$ (NA62) & Combined \\
\hline$\hat{c}, O\left(p^{4}\right)$, fit & $1.36 \pm 0.33_{\text {stat }} \pm 0.07_{\text {syst }}$ & $1.71 \pm 0.29_{\text {stat }} \pm 0.06_{\text {syst }}$ & $1.56 \pm 0.22_{\text {stat }} \pm 0.07_{\text {syst }}$ \\
$\hat{c}, O\left(p^{6}\right)$, fit & $1.67 \pm 0.39_{\text {stat }} \pm 0.09_{\text {syst }}$ & $2.21 \pm 0.31_{\text {stat }} \pm 0.08_{\text {syst }}$ & $2.00 \pm 0.24_{\text {stat }} \pm 0.09_{\text {syst }}$ \\
BR $O\left(p^{6}\right)$, fit & $(0.94 \pm 0.08) \times 10^{-6}$ & $(1.06 \pm 0.07) \times 10^{-6}$ & $(1.01 \pm 0.06) \times 10^{-6}$ \\
\hline
\end{tabular}

\section{Precision Lepton Universality Test}

In the SM, decays of pseudoscalar mesons to light leptons $P^{ \pm} \rightarrow l^{ \pm} v$, with $P=\pi, K, D, B$ (denoted as $\left.P_{l 2}\right)$ are helicity suppressed. While the predictions for single decay rates are affected by hadronic uncertainties, ratios of decay rates of the same parent meson do not depend on the decay constant due to cancellation effects and can be computed very precisely. In particular, the SM prediction for the ratio $R_{K}=\Gamma\left(K_{e 2}\right) / \Gamma\left(K_{\mu 2}\right)$, inclusive of internal bremsstrahlung radiation, is [6]

$$
R_{K}^{S M}=\frac{m_{e}^{2}}{m_{\mu}^{2}} \cdot\left(\frac{m_{K}^{2}-m_{e}^{2}}{m_{K}^{2}-m_{\mu}^{2}}\right)^{2} \cdot\left(1+\delta R_{Q E D}\right)=(2.477 \pm 0.001) \times 10^{-5},
$$

where $\delta R_{Q E D}=(3.79 \pm 0.04) \%$ is an electromagnetic correction. The factor $\left(m_{e} / m_{\mu}\right)^{2}$ accounts for the strong helicity suppression, which enhances the sensitivity to non-SM effects. Within extensions of the SM involving two Higgs doublets, the ratio $R_{K}$ is sensitive to lepton flavour violating effects originating at one-loop level from charged Higgs exchange [7]. This can lead to an enhancement of $R_{K}$ by $O(1 \%)$ [8], which is experimentally accessible.

The analysis strategy is based on counting the number of reconstructed $K_{e 2}$ and $K_{\mu 2}$ candidates collected simultaneously, i.e. the result does not rely on the absolute kaon flux, and several systematic effects cancel at first order. As the backgrounds and acceptances strongly depend on the momentum of the charged track, the analysis is performed independently in 10 bins of this variable and, in addition, for 4 samples with different data taking conditions, In each of the 40 bins, the ratio $R_{K}$ is computed:

$$
R_{K}=\frac{N\left(K_{e 2}\right)-N_{B}\left(K_{e 2}\right)}{N\left(K_{\mu 2}\right)-N_{B}\left(K_{\mu 2}\right)} \cdot \frac{A\left(K_{\mu 2}\right) \times f_{\mu} \times \epsilon\left(K_{\mu 2}\right)}{A\left(K_{e 2}\right) \times f_{e} \times \epsilon\left(K_{e 2}\right)} \cdot \frac{1}{f_{L K r}} \cdot \frac{1}{D},
$$

where $N\left(K_{l 2}\right)$ are the numbers of selected $K_{l 2}$ candidates $(l=e, \mu), N_{B}\left(K_{l 2}\right)$ are the numbers of background candidates, $f_{l}$ represent the particle $(e / \mu)$ ID efficiencies, $A\left(K_{l 2}\right)$ are the geometrical acceptances determined with MC simulations, $\epsilon\left(K_{l 2}\right)$ are the trigger efficiencies, $f_{L K r}$ is the global readout efficiency of the LKr, and $D$ is the downscaling factor of the $K_{\mu 2}$ trigger.
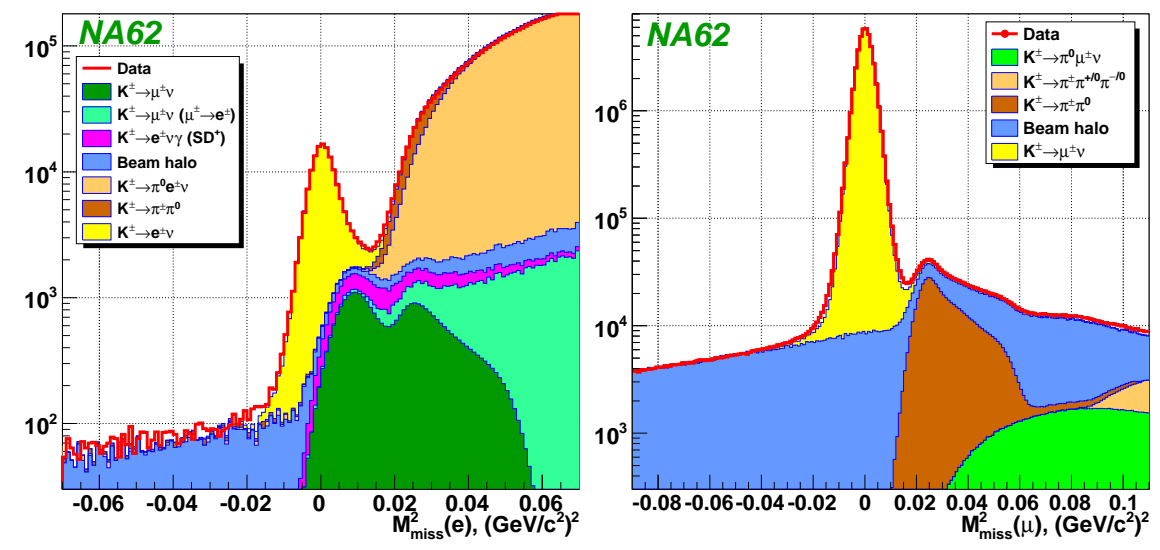

Fig. 2. Reconstructed squared missing mass distributions of the $K_{e 2}$ (left) and $K_{\mu 2}$ (right) candidates compared with the sum of normalized estimated signal and background components. 

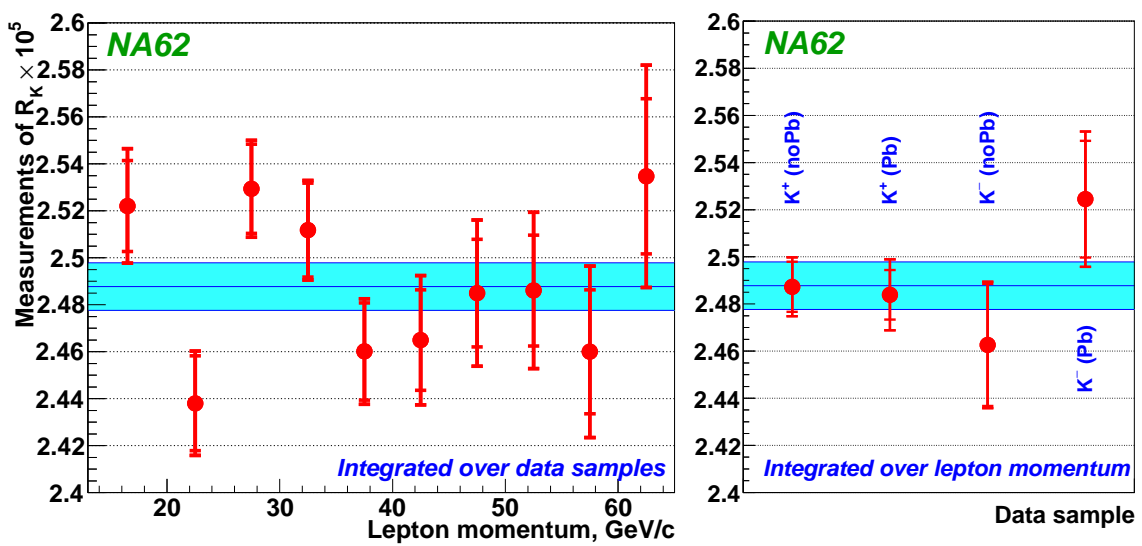

Fig. 3. Stability of the $R_{K}$ measurement versus lepton momentum (left) and for samples with different data taking conditions (right). The " $\mathrm{Pb} / \mathrm{noPb}$ " labels indicate that during the corresponding sample the lead wall was present/absent. The result of the combined fit over 40 data bins is shown by the horizontal band.

After a series of common selection criteria, the $K_{e 2} / K_{\mu 2}$ separation is achieved in two ways: a) Kinematical $K_{l 2}$ identification by reconstruction of the squared missing mass assuming the track to be an electron or a muon: $M_{\text {miss }}^{2}(l)=\left(P_{K}-P_{l}\right)^{2}$, where $P_{K}, P_{l}(l=e, \mu)$ are the kaon and lepton fourmomenta. However, a clear kinematical separation is possible only up to track momenta of $30 \mathrm{GeV} / c$. b) Particle identification by the ratio $\mathrm{E} / \mathrm{p}$ (= LKr energy deposit over track momentum), requiring $\mathrm{E} / \mathrm{p}<0.85$ for muons and $0.95<\mathrm{E} / \mathrm{p}<1.10$ for electrons. The particle IDs have very low inefficiencies ( $0.72 \%$ for electrons, a few $10^{-5}$ for muons).

After all cuts, $145,958 K_{e 2}$ and $4.2817 \times 10^{7} K_{\mu 2}$ candidates were selected, the latter collected with a pre-scaled trigger. The $K_{\mu 2}$ sample is almost background-free. The total background to the $K_{e 2}$ signal has been estimated with MC simulations and, where possible, with direct measurements to be (10.95 \pm $0.27) \%$, the main contribution coming from $K_{\mu 2}$ decays with a mis-identified muon. In very rare cases, the muon can deposit over $95 \%$ of its energy in the $\mathrm{LKr}$ calorimeter by emission of high energetic ('catastrophic') bremsstrahlung, thus faking an electron. The probability $P(\mu \rightarrow e)$ for this process (a few $10^{-6}$ ) has been measured directly as a function of momentum with a clean sample of muons (collected in separate periods of the 2007 data taking) passing a $\sim 9 X_{0}$ thick lead wall before hitting the LKr. Fig. 2 shows the reconstructed squared missing mass distributions of the $K_{e 2}$ and $K_{\mu 2}$ candidates compared with the sum of normalized MC signal and background components.

The result of the measurement, averaged over the 40 data bins and taking into account correlations between the systematic errors, is

$$
R_{K}=\left(2.488 \pm 0.007_{\text {stat }} \pm 0.007_{\text {syst }}\right) \times 10^{-5}=(2.488 \pm 0.010) \times 10^{-5} .
$$

The stability of the result is shown in Fig.3 as a function of lepton momentum and for the four different data samples $\left(K^{+}, K^{-}\right.$, with or without lead wall to measure $\left.P(\mu \rightarrow e)\right)$. The result is consistent with the SM prediction, and its $0.4 \%$ precision dominates the world average.

\section{The Ultra-Rare Decay $K^{+} \rightarrow \pi^{+} v \bar{v}$}

Among the flavour changing neutral current $K$ and $B$ decays, the $K \rightarrow \pi v \bar{v}$ decays represent golden channels to search for New Physics through the underlying mechanism of flavour mixing. These decays are extremely rare; in the SM, they are strongly suppressed due to the absence of tree-level contributions and the hard power-like GIM mechanism. On the other hand, the SM prediction is exceptionally precise: $\operatorname{BR}\left(K^{0} \rightarrow \pi^{0} v \bar{v}\right)=(2.43 \pm 0.39) \times 10^{-11}$ and $\mathrm{BR}\left(K^{+} \rightarrow \pi^{+} v \bar{v}\right)=(7.81 \pm 0.80) \times 10^{-11}$ [9] . The leading SM contribution is generated by top quark loops which can be computed with negligible theoretical uncertainty, and the hadronic matrix element can be extracted from the precisely measured 
$K^{ \pm} \rightarrow \pi^{0} e^{ \pm} v$ decay. Finally, the decays remain similarly predictive in various extensions of the SM, and even not large deviations from the SM value (e.g. 20\%) can be considered as a signal of New Physics. Furthermore, the study of $K^{+} \rightarrow \pi^{+} v \bar{v}$ allows the measurement of the CKM matrix element $\left|V_{t d}\right|$ complementary to those from $\mathrm{B}-\overline{\mathrm{B}}$ mixing and without requiring input from lattice QCD. Experimentally, only three $K^{+} \rightarrow \pi^{+} v \bar{v}$ events have been observed by the BNL 787/949 experiments, the result is $\operatorname{BR}\left(K^{+} \rightarrow \pi^{+} v \bar{v}\right)=\left(1.73_{-1.05}^{+1.15}\right) \times 10^{-10}$. However, only a measurement of the branching ratio with $\sim 10 \%$ accuracy can be a significant test of the SM. This precision is the main goal of the NA62 experiment [10]; it aims to collect about $100 \mathrm{~K}^{+} \rightarrow \pi^{+} v \bar{v}$ signal events in two years of data taking, keeping systematic uncertainties low and the background contamination at $\sim 10 \%$.

To reach this ambitious goal, various demanding requirements must be fulfilled. Assuming a $10 \%$ signal acceptance, the beam line must deliver at least $10^{13} \mathrm{~K}^{+}$decays. To keep the systematic uncertainty small requires a rejection of generic kaon decays by a factor of $10^{12}$ and the possibility to measure efficiencies and background suppression factors directly from data. The CERN-SPS extraction line, already used for NA48, can deliver the required intensity so that NA62 will be housed in the same experimental hall as NA48, located in the SPS North Area. Most of the NA48/2 apparatus, however, has to be replaced by new high performance detectors.

The concept of the experimental setup will be similar to NA48: We will use a decay in flight technique to identify $K^{+}$decay products from a $75 \mathrm{GeV} / c$ charged unseparated hadron beam with $1 \%$ momentum bite. A $\sim 100 \mathrm{~m}$ long beam line will form this secondary beam followed by a $\sim 80 \mathrm{~m}$ long evacuated decay volume and a series of downstream detectors to measure the decay products.

The signal signature is one track in the final state matched to one $K^{+}$track in the beam. The integrated rate upstream is about $800 \mathrm{MHz}$ (only $6 \%$ of the beam particles are kaons, the rest are pions and protons). The rate seen by the detector downstream is about $10 \mathrm{MHz}$, mainly from $K^{+}$decays. Very precise timing and excellent spatial resolution are required to match the upstream and downstream tracks. A beam tracker consisting of three silicon pixel stations with a time resolution of $\sim 100 \mathrm{ps}$ will deliver the upstream track information. Additionally, a differential Cherenkov counter on the beam line will identify the $K^{+}$in the beam. The background suppression profits from the high kaon beam momentum. Different techniques have to be combined to reach the necessary rejection power: 1) Kinematic rejection, requiring low mass and high efficiency spectrometers operating in vacuum. The downstream spectrometer will be composed of four straw chambers. 2) Precise timing, requiring various components with time resolutions of $\sim 100$ ps or below. 3) Excellent high efficiency photon and muon veto systems to largely suppress background from $K^{+} \rightarrow \pi^{+} \pi^{0}\left(\sim 10^{8}\right.$ rejection $)$ and $K^{+} \rightarrow \mu^{+} \nu\left(10^{11}\right.$ rejection). A full hermeticity for photons up to $50 \mathrm{mrad}$ will be provided by four subdetector systems: a series of annular lead glass calorimeters surrounding the decay and detector volume covering the large angle range, the NA48 LKr calorimeter for the forward region, and two small angle calorimeters providing coverage for photons emitted at close to zero angles to the beam. 4) Precise particle identification systems to distinguish $\pi^{+}, \mu^{+}$and positrons. Downstream, a RICH detector will provide the pion/muon separation and define the event time due to its excellent time resolution.

The design of the experimental apparatus and the R\&D of the new detector components have been completed. The first technical run is scheduled for October-December 2012. The main data taking will start in 2014 after the long LHC shutdown.

\section{References}

1. V. Fanti et al., Nucl. Instrum. Methods A574, (2007) 433.

2. M. Antonelli et al. (Flavianet Kaon WG), E. Phys. J. C69, (2010) 399.

3. G. Ecker, A. Pich and E. de Rafael, Nucl. Phys. B303, (1988) 665.

4. G. D'Ambrosio and J. Portolés, Phys. Lett. B386, (1996) 403.

5. P. Kitching et al., Phys. Rev. Lett. 79, (1997) 4079.

6. V. Cirigliano and I. Rosell, Phys. Rev. Lett. 99, (2007) 231801.

7. A. Masiero, P. Paradisi, R. Petronzio, Phys. Rev. D74, (2006) 011701; JHEP 0811, (2008) 42.

8. J. Girrbach and U. Nierste, arXiv:1202.4906.

9. J. Brod, M. Gorbhahn, E. Stamou, Phys. Rev. D83, (2011) 034030.

10. G. Anelli et al., "Proposal to measure the rare decay $K^{+} \rightarrow \pi^{+} v \bar{v}$ at the CERN SPS", CERNSPSC-2005-013, SPSC-P-326. 